\title{
Courtage de connaissances sur les maladies infectieuses pour la santé publique
}

\author{
Margaret Haworth-Brockman ${ }^{1,2 \star}$, Yoav Keynan ${ }^{1,2,3}$
}

\section{Résumé}

Les Centres de collaboration nationale (CCN) en santé publique (CCNSP) ont été établis en 2005 dans le cadre de l'engagement du gouvernement fédéral à renouveler et à renforcer la santé publique à la suite de l'épidémie du syndrome respiratoire aigu sévère (SRAS). Ils ont été mis sur pied pour appuyer l'application des connaissances en vue d'une utilisation plus opportune de la recherche scientifique et d'autres connaissances dans les pratiques, les programmes et les politiques de santé publique au Canada. Six centres composent les CCNSP, dont le Centre de collaboration nationale des maladies infectieuses (CCNMI). Le CCNMI collabore avec des professionnels de la santé publique pour trouver, comprendre et utiliser des recherches et des données probantes sur les maladies infectieuses et les déterminants de la santé associés. Le CCNMI a pour mandat de forger des liens entre ceux qui génèrent et ceux qui utilisent les connaissances sur les maladies infectieuses.

À titre de premier article d'une série sur le CCNSP, nous décrivons notre rôle dans le courtage de connaissances et les nombreuses méthodes et produits que nous avons élaborés. De plus, nous illustrons comment le CCNMI a été en mesure de travailler avec la santé publique pour générer et échanger des connaissances pendant la pandémie du coronavirus de 2019 (COVID-19).
Cette oeuvre est mise à la disposition selon les termes de la licence internationale Creative Commons Attribution 4.0

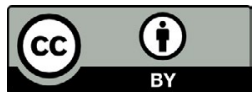

Affiliations

${ }^{1}$ Centre de collaboration nationale des maladies infectieuses, Faculté des sciences de la santé Rady, Université du Manitoba, Winnipeg, MB

${ }^{2}$ Département des sciences de la santé communautaire, Collège de médecine Max Rady, Université du Manitoba, Winnipeg, MB

${ }^{3}$ Départements de médecine interne et de microbiologie médicale, Collège de médecine Max Rady, Université du Manitoba, Winnipeg, MB

\section{*Correspondance :}

haworth-brockman@umanitoba.ca pour la santé publique. Relevé des maladies transmissibles au Canada 2021;47(3):175-80. https://doi.org/10.14745/ccdr.v47i03a06f

Mots-clés : santé publique, maladies infectieuses, courtage de connaissances, Canada, COVID-19

\section{Introduction}

Les Centres de collaboration nationale (CCN) de la santé publique (CCNSP) ont été établis en 2005 dans le cadre de I'engagement du gouvernement fédéral du Canada à renouveler et à renforcer la santé publique à la suite de l'épidémie du syndrome respiratoire aigu sévère (SRAS). Les CCN ont été créés pour appuyer l'application des connaissances afin d'utiliser plus rapidement la recherche scientifique et d'autres connaissances dans les pratiques, les programmes et les politiques de santé publique au Canada (1). Financé par l'Agence de la santé publique du Canada (l'Agence), chacun des six CCN est hébergé par une université ou un organisme gouvernemental et se concentre sur un domaine spécifique de la santé publique : Déterminants de la santé, Santé environnementale, Politiques publiques et santé, Santé autochtone, Maladies infectieuses et Méthodes et outils d'application des connaissances (1).

Le Centre de collaboration nationale des maladies infectieuses (CCNMI) est hébergé par l'Université du Manitoba et travaille avec des professionnels de la santé publique pour trouver, comprendre et utiliser la recherche et les données probantes sur les maladies infectieuses et les déterminants sous-jacents qui influent sur la distribution et l'impact des maladies et sur les stratégies d'atténuation efficaces. Nos huit employés établissent des liens entre ceux qui génèrent et ceux qui utilisent les connaissances sur les maladies infectieuses liées à un large éventail de sujets, y compris la résistance et la gestion des antimicrobiens, les infections transmissibles sexuellement et par le sang (ITSS), les maladies évitables par la vaccination, la tuberculose et les infections émergentes.

En tant que premier article d'une série sur le CCNSP, nous décrivons le rôle de l'application des connaissances, en particulier en tant que courtiers de connaissances $(2,3)$, nos nombreuses méthodes et nombreux produits, puis nous illustrons comment le CCNMI a été en mesure de travailler avec les représentants de la santé publique pour mobiliser avec agilité et réactivité les connaissances pendant la pandémie de coronavirus 2019 (COVID-19). 


\section{Cadre conceptuel de la science de programmes pour le courtage de connaissances}

Chaque année, le CCNMI entreprend une variété de projets en se fondant sur des consultations avec les intervenants et sur les preuves des lacunes de connaissances existantes. Les événements et les ressources sont élaborés en consultation avec des partenaires de partout au Canada, bien qu'ils soient souvent adaptés à des publics particuliers ou à des contextes régionaux. Dans la mesure du possible, nous travaillons avec les autres CCN pour assurer une plus grande applicabilité et pertinence.

Le CCNMI utilise le cadre conceptuel de la science de programmes pour organiser son travail et se concentrer sur les étapes d'interventions en santé publique. La science des programmes est une application systématique des connaissances scientifiques théoriques et empiriques pour améliorer la conception, la mise en œuvre et l'évaluation des programmes de santé publique (4). Elle permet d'obtenir un engagement rigoureux à comprendre les différents types de données probantes qui sont nécessaires et auxquelles on peut donner suite dans des contextes particuliers (5). Ce cadre nous permet de démontrer l'interdépendance des preuves liées aux politiques et aux pratiques dans différents domaines, tout en mettant l'accent sur le contexte et les circonstances des pratiques prometteuses dans trois domaines : 1) les facteurs et le fardeau des maladies infectieuses, en relation avec le domaine de la surveillance dans la science des programmes; 2 ) les réponses et les interventions de santé publique, en relation avec le même domaine dans la science des programmes; et 3) les systèmes et les politiques de surveillance des maladies infectieuses, en relation avec le domaine de la surveillance et de l'évaluation dans la science des programmes (6) (tableau 1). Ce faisant, nous illustrons les approches globales qui s'appliquent à plusieurs maladies et les résultats souhaités en matière de santé publique, surtout en ce qui concerne les approches d'équité en santé pour les syndémies et les populations défavorisées.

Le courtage de connaissances est à la fois un processus et un produit $(7,8)$. Le CCNMI entreprend diffèrent types de projets dans les trois domaines scientifiques de programme (figure 1). Le premier type de projet consiste à créer et à favoriser l'échange de connaissances entre le personnel de la santé publique à tous les niveaux, et à organiser des webinaires, des présentations en groupe, des ateliers et des rassemblements. Le CCNMI réunit des experts de la communauté, des politiques, des cliniques et du milieu universitaire de plusieurs administrations pour discuter d'enjeux et échanger des stratégies de santé publique réussies (et celles qui présentent des défis). Les conversations facilitées par le CCNMI encouragent l'examen réfléchi de questions opportunes. L'utilisation de formats plus récents, comme les discussions en cercles restreints (9), les commentaires d'experts et les séminaires préparatoires, permet aux présentateurs et aux participants d'avoir plus de temps pour tenir des discussions animées sur le contenu.
Tableau 1 : Exemples de domaines de courtage de connaissances du Centre de collaboration nationale des maladies infectieuses dans un cadre scientifique de programme

\begin{tabular}{|c|c|c|}
\hline $\begin{array}{l}\text { Domaines } \\
\text { scientifiques de } \\
\text { programme }\end{array}$ & $\begin{array}{l}\text { Sujets de courtage de } \\
\text { connaissances }\end{array}$ & $\begin{array}{l}\text { Résultats } \\
\text { attendus }\end{array}$ \\
\hline $\begin{array}{l}\text { Facteurs et fardeau } \\
\text { des maladies } \\
\text { infectieusess }\end{array}$ & $\begin{array}{l}\text { - Facteurs et fardeau des } \\
\text { maladies particulières } \\
\text { - Facteurs et fardeau dans } \\
\text { certaines populations } \\
\text { - Preuves de surveillance }\end{array}$ & $\begin{array}{l}\text { CHOISIR } \\
\text { - } \text { Meilleure } \\
\text { stratégie } \\
\text { - Populations } \\
\text { - } \text { appropriées } \\
\text { - } \text { Bon moment }\end{array}$ \\
\hline $\begin{array}{l}\text { Interventions et } \\
\text { réponse en santé } \\
\text { publique }\end{array}$ & $\begin{array}{l}\text { - Interventions } \\
\text { appropriées pour la TB, } \\
\text { les ITSS, la RAM, etc. } \\
\text { - Santé publique pour les } \\
\text { populations mobiles } \\
\text { - Études de cas } \\
\text { prometteuses sur la } \\
\text { réduction des méfaits } \\
\text { - Tests au point de service } \\
\text { - Améliorer la confiance } \\
\text { dans les vaccins }\end{array}$ & $\begin{array}{l}\text { FAIRE } \\
\text { - Les bonnes } \\
\text { choses } \\
\text { - De la bonne } \\
\text { façon }\end{array}$ \\
\hline $\begin{array}{l}\text { Surveillance et } \\
\text { évaluation }\end{array}$ & $\begin{array}{l}\text { - Utilisations des } \\
\text { mégadonnées en santé } \\
\text { publique } \\
\text { - Indicateurs de } \\
\text { rendement des } \\
\text { programmes sur la TB } \\
\text { pour améliorer l'équité } \\
\text { - Ressources de } \\
\text { surveillance de la RAM }\end{array}$ & $\begin{array}{l}\text { ASSURER } \\
\text { - Mise à l'échelle } \\
\text { appropriée } \\
\text { - Efficacité } \\
\text { - Changement, } \\
\text { au besoin }\end{array}$ \\
\hline
\end{tabular}

Figure 1 : Projets de courtage de connaissances du Centre de collaboration nationale des maladies infectieuses, par type et année, 2015-2020

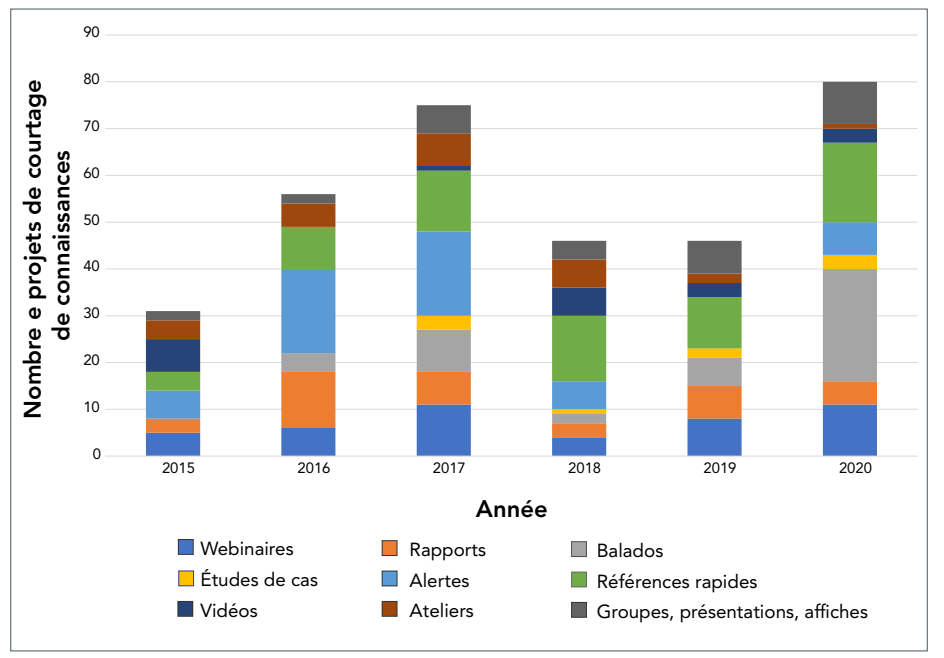


Le CCNMI élabore et diffuse de nouveaux produits de connaissances qui appliquent les données probantes à des contextes précis de pratiques et de politiques de santé publique. Ces produits de connaissances comprennent des balados, des vidéos animées et des études de cas en langage simple, ainsi que des revues et des articles de revue plus traditionnels, réalistes, de portée pertinente et narratifs. Nous adaptons les produits de connaissances pour répondre aux besoins particuliers des infirmières, des médecins hygiénistes, des analystes des politiques, des étudiants et des fournisseurs de première ligne en santé publique.

Le CCNMI a intégré trois grandes priorités pour de sujets liés aux maladies. La première priorité est de mettre l'accent sur la mobilité des populations au Canada. Des projets antérieurs sur les approches en matière de santé publique pour les réfugiés et les demandeurs d'asile, ainsi que sur les collectivités évacuées en raison d'incendies et d'inondations (10), ont mis en évidence le besoin de courtage de connaissances sur les effets de la migration au Canada et à l'intérieur du pays. Par exemple, la collecte de données sur les éclosions de tuberculose et de syphilis ainsi que leur gestion sont compliquées lorsque les patients doivent déménager (11), y compris des régions rurales vers les villes (12). Les syndémies des ITSS et de la tuberculose, combinées à des épidémies croissantes d'utilisation d'opioïdes et de méthamphétamine en cristaux, sont d'autant plus compliquées par les déplacements, I'incarcération et les divisions entre les provinces et les territoires $(13,14)$.

La deuxième priorité du $\mathrm{CCNMI}$ est de répondre aux inégalités dans les interventions en matière de santé publique à l'égard des maladies transmissibles dans les collectivités rurales et éloignées. Bien que les ressources soient mises à rude épreuve dans tous les bureaux de santé publique, cela est particulièrement vrai à l'extérieur des principaux centres urbains. En plus de travailler avec le personnel de la santé publique pour comprendre les facteurs particuliers des maladies infectieuses dans les régions rurales, éloignées et nordiques, y compris les facteurs associés à la stigmatisation et à la mauvaise santé mentale, le CCNMI sert de secrétariat au Réseau de santé publique des régions rurales, éloignées et nordiques, composé de médecins de santé publique, et s'associe à des universitaires et à des autorités sanitaires autochtones pour élaborer des approches propres aux Premières Nations, aux Métis et aux Inuits pour lutter contre la tuberculose, les ITSS et les maladies à transmission vectorielle.

La troisième priorité du CCNMI est d'appuyer les possibilités d'utiliser les mégadonnées pour la surveillance, la prévention, le contrôle et le suivi des maladies infectieuses. Le CCNMI a été à l'avant-garde de la création d'occasions d'échange de connaissances entre les modélisateurs mathématiques et le personnel de la santé publique $(15,16)$. Nous avons récemment entrepris de nouvelles collaborations avec d'éminents experts-conseils canadiens en mégadonnées pour aider à démontrer comment les mégadonnées peuvent être utilisées pour planifier et évaluer les interventions en santé publique.
L'utilisation du cadre conceptuel de la science de programmes permet au CCNMI d'appliquer des méthodes et des approches de courtage de connaissances dans plusieurs domaines. Par exemple, le CCNMI indique clairement quelles collectivités sont désavantagées (e.g. en raison de leur emplacement géographique, du racisme systémique et historique ou des services de santé et de santé publique inadéquats ou insuffisants) et quelles inégalités peuvent être atténuées pour réduire le fardeau de la maladie. Dans le domaine scientifique des interventions en santé publique, nous soulignons les pratiques prometteuses utilisées pour contrôler une maladie à un endroit précis qui peuvent être adaptées pour répondre à une autre (e.g. fournir des données probantes sur les interventions rapides pour réduire les éclosions de $\mathrm{VIH}$ en Indiana qui peuvent être adaptées pour répondre à la montée de l'hépatite $C$ dans les provinces des Prairies canadiennes) (17). Dans le domaine du suivi et de l'évaluation, les projets du CCNMI qui encouragent I'utilisation des indicateurs ventilés et croisés pour la surveillance du rendement des programmes de santé publique (18) ont été adaptés pour appuyer l'intégration de l'équité en santé dans les organisations de santé publique (19).

\section{Le Centre de collaboration nationale des maladies infectieuses dans le contexte de la COVID-19}

Avec des données probantes disponibles au début de janvier 2020 sur la COVID-19 qui était susceptible d'être transmise au-delà de l'Asie, le CCNMI a mis au point une nouvelle ressource de liens rapides pour le personnel de la santé publique, rassemblant des renseignements clés de l'Organisation mondiale de la Santé, de l'Agence et des Centers for Disease Control and Prevention des États-Unis. Des descriptions plus détaillées ont été transformées en compte rendu de maladie et affichées en ligne une semaine plus tard. Le résumé de I'information a été mis à jour tout au long de 2020 pour suivre l'évolution des connaissances cliniques et épidémiologiques liées à la pandémie.

À la fin de janvier 2020, il était clair que la nouvelle maladie allait nécessiter plus d'attention de la part de la santé publique, tant au Canada qu'ailleurs dans le monde. Le CCNMI a rapidement lancé une série de balados sur de nombreux aspects importants de la COVID-19, fournissant aux publics de la santé publique de brèves réponses aux questions fréquemment posées et résumant les plus récentes données probantes d'experts de partout au Canada. II y a maintenant 20 épisodes de baladodiffusion disponibles pour les médecins, les infirmières, les inspecteurs sur le terrain et les analystes des politiques de la santé publique qui ont été téléchargés plus de 1200 fois jusqu'à maintenant et qui ont été classés parmi les 30 meilleures séries de balados sur la santé publique par MPH Online, une ressource en ligne indépendante pour les étudiants en santé publique. 
La souplesse des ententes entre le CCNMI et l'Agence nous a permis d'offrir plusieurs projets sur la COVID-19 et d'y donner suite tout au long de 2020. Ces projets comprenaient le soutien au courtage de connaissances par l'entremise de nouvelles subventions des Instituts de recherche en santé du Canada (huit subventions à ce jour), la création d'un centre pour les lignes directrices du Réseau des laboratoires de santé publique du Canada, ainsi que l'élaboration d'une série de webinaires visant à présenter les concepts de modélisation mathématique à des publics de santé publique et à approfondir la manière dont les modèles sont utilisés pour planifier les mesures liées à la COVID-19 (plus de 350 participants). De plus, le CCNMI a mis en contact des experts canadiens en modélisation avec des collègues à Medellín, en Colombie, pour appuyer leur modélisation continue en matière de santé publique. À I'hiver 2020 à 2021, le CCNMI a coanimé les webinaires d'information de l'Agence sur les nouveaux vaccins contre la COVID-19 (plus de 5000 participants).

Le personnel et les étudiants du CCNMI mènent des projets à plus long terme concernant la migration des populations et les préoccupations liées à l'équité dans les régions rurales, éloignées et nordiques. Ces projets comprennent une analyse à venir des considérations d'équité des processus décisionnels en matière de traitement clinique et l'élaboration de nouveaux modèles pour prédire les résultats à long terme des fermetures d'écoles (20) et des mesures d'isolement dans les établissements de soins de longue durée.

\section{Contributions aux compétences en santé publique dans le domaine des maladies infectieuses}

Les activités du CCNMI s'harmonisent de plusieurs façons avec les principaux domaines d'intérêt au sein du système de santé publique du Canada. Le CCNMI contribue à l'objectif global de l'administratrice en chef de la santé publique qui consiste à " mettre tout le monde sur un pied d'égalité » (21) en matière de prévention et de contrôle de la COVID-19, de la tuberculose, des ITSS et de la résistance aux antimicrobiens en favorisant les mesures sur les déterminants de la santé et en renforçant les partenariats multisectoriels. Le CCNMI encourage le partage intergouvernemental d'approches éprouvées et fructueuses pour atteindre les populations mal desservies.
Grâce à nos partenariats universitaires et de santé publique, nous disposons de possibilités d'enseignement et de mentorat pour les étudiants, en particulier pour les compétences essentielles suivantes (22):

- Prévention et contrôle des maladies infectieuses

- Interventions d'urgence

- Évaluation, analyse et planification de programme

Les étudiants de premier et de deuxième cycles en santé publique, en médecine, en sciences fondamentales, en sciences infirmières, en communications et en sociologie comptent parmi les plus de 40 stagiaires qui ont acquis de nouvelles compétences en courtage de connaissances au CCNMI (figures 2 et 3 ). Au fil du temps, le CCNMI a également attiré des participants d'autres secteurs pour encourager l'échange de connaissances afin d'améliorer les interventions en santé publique pour la prévention et le contrôle des maladies infectieuses.

Figure 2 : Nombre d'étudiants au Centre de collaboration nationale des maladies infectieuses, par sujet de recherche, sexe et année, 2015-2020

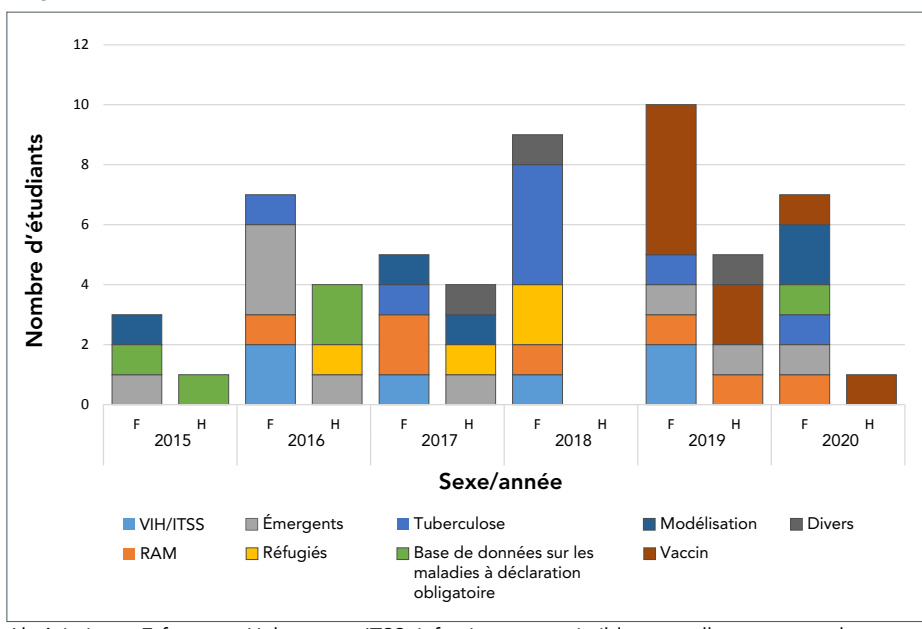

Abréviations : $\mathrm{F}$, femmes; $\mathrm{H}$, hommes; ITSS, infections transmissibles sexuellement et par le sang: $\mathrm{RAM}$, résistance aux antimicrobiens; $\mathrm{VIH}$, virus de l'immunodéficience humaine

Figure 3 : Nombre d'étudiants au Centre de collaboration nationale des maladies infectieuses, par universités d'attache, sexe et année, 2015-2020

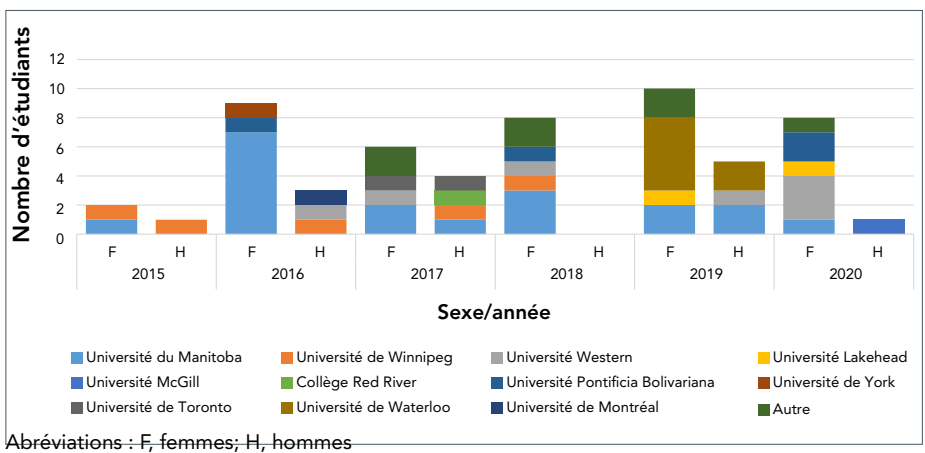




\section{Conclusion}

Un courtier de connaissances s'adapte aux changements sociaux et techniques de chaque situation et établit un processus unique et pertinent pour créer des relations et promouvoir I'apprentissage et le changement (23). Cette description décrit bien le rôle du CCNMI. L'analyse de l'augmentation de la portée, de l'adoption et de l'incidence de nos activités et de nos produits au fil des ans confirme que notre approche a de la valeur pour les publics de la santé publique au Canada. En travaillant avec les autres CCN et entre les disciplines, les secteurs et les paliers de gouvernement, le CCNMI optimise la collecte et la diffusion des connaissances ainsi que leur mobilisation, il facilite l'établissement de réseaux et de partenariats et attire l'attention sur les lacunes et les problèmes de connaissances pour les populations mal desservies. Notre capacité de contribuer aux sujets tels que le logement et la toxicomanie est essentielle pour répondre aux déterminants qui sous-tendent souvent la transmission de la maladie.

\section{Déclaration des auteurs}

M. H. B. - Conceptualisation et rédaction des versions préliminaires, révisions finales Y. K. - Conceptualisation, données de fond, examen des versions préliminaires

\section{Intérêts concurrents}

Aucun.

\section{Remerciements}

Nous remercions nos collègues du Programme des Centres de collaboration nationale en santé publique et les rédacteurs de nous avoir donné cette occasion.

\section{Financement}

Ce travail a été financé par l'Agence de la santé publique du Canada.

\section{Références}

1. Medlar B, Mowat D, Di Ruggiero E, Frank J. Introducing the National Collaborating Centres for Public Health. CMAJ 2006;175(5):493-4. DOI PubMed

2. Centre de collaboration nationale des maladies infectieuses. Le courtage des connaissances au service des maladies infectieuses en santé publique au Canada. Winnipeg (MB) : CCNMI; 2014. https://ccnmi.ca/publications/le-courtag e-des-connaissances-au-service-des-maladies-infectieuses -en-sante-publique-au-canada/
3. Dobbins M, Robeson P, Ciliska D, Hanna S, Cameron R, O'Mara L, DeCorby K, Mercer S. A description of a knowledge broker role implemented as part of a randomized controlled trial evaluating three knowledge translation strategies. Implement Sci 2009;4:23. DOI PubMed

4. Blanchard JF, Aral SO. Program Science: an initiative to improve the planning, implementation and evaluation of $\mathrm{HIV} /$ sexually transmitted infection prevention programmes. Sex Transm Infect 2011;87(1):2-3. DOI PubMed

5. Aral SO, Blanchard JF. The Program Science initiative: improving the planning, implementation and evaluation of HIV/STI prevention programs. Sex Transm Infect 2012;88(3):157-9. DOl PubMed

6. Becker M, Haworth-Brockman M, Keynan Y. The value of program science to optimize knowledge brokering on infectious diseases for public health. BMC Public Health 2018;18(1):567. DOI PubMed

7. Pablos-Mendez A, Shademani R. Knowledge translation in global health. J Contin Educ Health Prof 2006;26(1):81-6. DOI PubMed

8. Green LW, Ottoson JM, García C, Hiatt RA. Diffusion theory and knowledge dissemination, utilization, and integration in public health. Annu Rev Public Health 2009;30(1):151-74. DOI PubMed

9. Facing History and Ourselves. Teaching Strategy: Fishbowl (accédé 2020-10-02). https://www.facinghistory.org/ resource-library/teaching-strategies/fishbowl

10. Clow B, Haworth-Brockman M, Boily-Larouche G, Qadar Z, Keynan Y. Looking for evidence of public health's role for long-term evacuees. Front Public Health 2019;7:15. DOI PubMed

11. Asadi L, Heffernan C, Menzies D, Long R. Effectiveness of Canada's tuberculosis surveillance strategy in identifying immigrants at risk of developing and transmitting tuberculosis: a population-based retrospective cohort study. Lancet Public Health 2017;2(10):e450-7. DOI PubMed

12. Orr P. Adherence to tuberculosis care in Canadian Aboriginal populations, Part 1: definition, measurement, responsibility, barriers. Int J Circumpolar Health 2011;70(2):113-27. DOI PubMed

13. Quinn B, Gorbach PM, Okafor CN, Heinzerling KG, Shoptaw S. Investigating possible syndemic relationships between structural and drug use factors, sexual HIV transmission and viral load among men of colour who have sex with men in Los Angeles County. Drug Alcohol Rev 2020;39(2):116-27. DOl PubMed

14. Wise A, Finlayson T, Sionean C, Paz-Bailey G. Incarceration, HIV risk-related rehaviors, and partner characteristics among heterosexual men at increased risk of HIV infection, 20 US cities. Public Health Rep 2019;134(1_suppl):63S-70S. DOI PubMed 
15. Moghadas SM, Haworth-Brockman M, Isfeld-Kiely H, Kettner J. Improving public health policy through infection transmission modelling: Guidelines for creating a Community of Practice. Can J Infect Dis Med Microbiol 2015;26(4):191-5. DOI PubMed

16. Milwid R, Steriu A, Arino J, Heffernan J, Hyder A, Schanzer D, Gardner E, Haworth-Brockman M, Isfeld-Kiely H, Langley JM, Moghadas SM. Toward standardizing a lexicon of infectious disease modeling terms. Front Public Health 2016;4:213. DOI PubMed

17. National Collaborating Centre for Infectious Diseases. Harm Reduction in a Rural Setting: Lessons learned from HCV and HIV outbreaks in Scott County, Indiana. Winnipeg (MB): NCCID; 2020 (accédé 2020-12-14). https://nccid.ca/ publications $/$ harm-reduction-in-a-rural-setting $/$ ?hilite $=\% 27 \mathrm{~h}$ arm\%27

18. Haworth-Brockman MJ, Keynan Y. Strengthening tuberculosis surveillance in Canada. CMAJ 2019;191(26):E743-4. DOI PubMed

19. Centre de collaboration nationale des maladies infectieuses. Mesurer ce qui compte en plein cœur de la pandémie de COVID-19 - Indicateurs d'équité pour la santé publique. Winnipeg (MB) : CCNMI; 2020. https:// ccnmi.ca/publications/mesurer-ce-qui-compte-en-plei n-coeur-de-la-pandemie-de-covid-19-indicateurs-dequitepour-la-sante-publique//
20. Abdollahi E, Haworth-Brockman M, Keynan Y, Langley JM, Moghadas SM. Simulating the effect of school closure during COVID-19 outbreaks in Ontario, Canada. BMC Med 2020;18(1):230. DOI PubMed

21. Agence de la santé publique du Canada. Approche de l'administrateur en chef de la santé publique du Canada en matière d'équité en santé et domaines d'intérêt. Ottawa (ON) : ASPC; 2020. https://www.canada.ca/fr/ sante-publique/organisation/structure-organisationnelle/ administrateur-chef-sante-publique-canada/ declarations-administrateur-chef-sante-publique-canada/ approche-matiere-equite-sante.html

22. Agence de la santé publique du Canada. Compétences essentielles en santé publique au Canada version 1.0. Ottawa (ON) : ASPC; 2008. https://www.phac-aspc.gc.ca/ php-psp/ccph-cesp/pdfs/zcard-fra.pdf

23. Conklin J, Lusk E, Harris M, Stolee P. Knowledge brokers in a knowledge network: the case of Seniors Health Research Transfer Network knowledge brokers. Implement Sci 2013;8:7. DOI PubMed 